\title{
A Novel Hybrid Optimization Algorithm and its Application in Solving Complex Problem
}

\author{
Hao Jia ${ }^{1,2}$ \\ ${ }^{1}$ Department of Electrical Engineering, Dalian Institute of Science and Technology, \\ Dalian 116052 China \\ ${ }^{2}$ The Provincial Key Laboratory for Computer Information Processing Technology, \\ Soochow University, suzhou 215006 China \\ jiangdao1979@yeah.net
}

\begin{abstract}
Ant colony optimization (ACO) algorithm is a new heuristic algorithm which has been demonstrated a successful technology and applied to solving complex optimization problems. But the ACO exists the low solving precision and premature convergence problem, particle swarm optimization (PSO) algorithm is introduced to improve performance of the ACO algorithm. A novel hybrid optimization (HPSACO) algorithm based on combining collaborative strategy, particle swarm optimization and ant colony optimization is proposed for the traveling salesman problems in this paper. The HPSACO algorithm makes use of the exploration capability of the PSO algorithm and stochastic capability of the ACO algorithm. The main idea of the HPSACO algorithm uses the rapidity of the PSO algorithm to obtain a series of initializing optimal solutions for dynamically adjusting the initial pheromone distribution of the ACO algorithm. Then the parallel search ability of the he ACO algorithm are used to obtain the optimal solution of solving problem. Finally, various scale TSP are selected to verify the effectiveness and efficiency of the proposed HPSACO algorithm. The simulation results show that the proposed HPSACO algorithm takes on the better search precision, the faster convergence speed and avoids the stagnation phenomena.
\end{abstract}

Keywords: ant colony optimization; particle swarm optimization; hybrid optimization algorithm; traveling salesman problem; stochastic capability

\section{Introduction}

The traveling salesman problem (TSP) [1] is one of the most important combinatorial problems, which is representative of a large number of scientific and engineering problems. The TSP is a traveling salesman to travel all cities but each city can only be visited once. Its task is to determine a route among a given set of nodes with the shortest possible length by the salesman. In many fields, TSP and its variants are good ground for testing optimization techniques. These researchers proposed simulated annealing, tabu search, evolutionary method, neural network, genetic algorithm, particle swarm optimization, ant colony optimization and bee colony algorithm and so on to solve all aspects of combinatorial optimization problems and benchmark problems in order to obtain the optimal solutions in recent years [2-6].

ACO [7] is known as the ant system which was proposed by Dorigo in 1997. It has received increased interests from researchers to solve complex optimization problems in large amount of actual engineering in recent years. But the ACO algorithm exists the itself shortcomings in the application, such as the long calculating time, stagnation phenomenon, 
uniform distribution pheromone and so on. So many improved algorithms based on the ACO are proposed. Stutzle and Hoos [8] proposed a MAX-MIN ant system and local search to solve the traveling salesman problem. Lee et al. [9] proposed an immunity based ACO algorithm for solving weapon-target assignment problems. Zecchin et al. [10] proposed a formulation of ant algorithms for the water distribution system optimization. Ho and Yang [11] proposed a computationally efficient vector optimizer using ant colony optimizations algorithm for multobjective designs. Chang et al. [12] proposed an ant algorithm for balanced job scheduling in grids. Meena et al. [13] formulated the text feature selection problem as a combinatorial problem and proposed an Ant Colony Optimization (ACO) algorithm to find the nearly optimal solution for the same. Shima and Hossein [14] proposed a novel feature selection (ABACO) algorithm based on ant colony optimization (ACO) for feature subset selection.

With the rapid development of the science and technology, the complex optimization problems are becoming too complicated to obtain the much satisfied results by using the basic ACO algorithm and improved ACO algorithm. So more and more hybrid optimization algorithms by utilizing several optimization algorithms have received the attention. In this paper, a novel hybrid optimization (HPSACO) algorithm based on combining collaborative strategy, particle swarm optimization and ant colony optimization is proposed to solve the complex optimization problems. The HPSACO algorithm can obtain better optimization solutions by exploiting the complementary characteristics of different optimization strategies and algorithms. It takes advantage of the merits of the PSO algorithm and ACO algorithm to solve the complex optimization problem in order to fully reflect the whole optimization ability of the HPSACO algorithm, improve the initial pheromone distribution of the ACO algorithm.

The rest of the paper is organized as follows. Section 2 briefly describes the particle swarm optimization and ant colony optimization algorithms. Section 3 discusses a novel hybrid optimization algorithm named HPSACO algorithm in details. Section 4 tests the HPSACO algorithm on TSP, and the results obtained are presented and discussed. Finally, conclusions are given in section 5 .

\section{Particle Swarm Optimization and Ant Colony Optimization Algorithms}

\subsection{Particle Swarm Optimization Algorithm}

The PSO algorithm [2] is a population-based search algorithm based on the simulation of the social behavior of birds within a flock [2]. In PSO algorithm, individuals, referred to as particles, are "flown" through hyper dimensional search space. The particles' positions within the search space are changed based on the social-psychological tendency of individuals in order to delete the success of other individuals. The changing of one particle within the swarm is influenced by the experience, or knowledge. The consequence of modeling for this social behavior is that the search is processed in order to return toward previously successful regions in the search space. Namely, the velocity $(v)$ and position $(x)$ of each particle will be changed by the particle best value $(p B)$ and global best value $(g B)$. The velocity and position updating of the particle is shown by the followed expression:

$$
\begin{aligned}
& v_{i j}(t+1)=w v_{i j}(t)+c_{1} r_{1}\left(p B_{i j}(t)-x_{i j}(t)\right)+c_{2} r_{2}\left(g B_{i j}(t)-x_{i j}(t)\right) \\
& x_{i j}(t+1)=x_{i j}(t)+v_{i j}(t+1)
\end{aligned}
$$


Where $v_{i j}(t+1)$, velocities of particle $i$ at iterations $j, x_{i j}(t+1)$, positions of particle $i^{\text {th }}$ at iterations $j^{t h}$. $w$ is inertia weight to be employed to control the impact of the previous history of velocities. Accordingly, the parameter $w$ regulates the trade-off between the global and local exploration abilities of the swarm.

\subsection{Ant Colony Optimization Algorithm}

Ant colony algorithm (ACO) was introduced by Marco Dorigo [7]. It is a branch of newly developed form of artificial intelligence called swarm intelligence, which studies "the emergent collective intelligence of groups of simple agents". The ACO is a metaheuristic inspired by the behavior of real ants in their search for the shortest path to food. When ants move, ants will leave a chemical pheromone trail on the ground. The ACO simulates the techniques employed by real ants to rapidly establish the shortest route from a food source to their nest and vice versa (adapting to changes in the environment) without the use of visual information. The basic idea of the ACO is to model the problem that is being solved, just as the search for a minimum cost path in a graph that uses artificial ants to search for good paths. Artificial ants walk through this graph to look for good paths. Since the ants have a rather simple behavior, they can only find poor-quality paths on their own. Better paths are found as the emergent result of the global cooperation among ants.

The ACO consists of a number of iterations of solution construction. In each iteration, a number of ants construct complete solutions by using heuristic information and the collected experiences of the previous groups of ants. These collected experiences are represented by the pheromone trail which is deposited on the constituent elements of a solution. Small quantities are deposited during the construction phase while larger amounts are deposited at the end of each iteration in proportion to solution quality. Pheromone can be deposited on the components and/or the connections used in a solution depending on the problem. In the ACO algorithm, the ACO simulates the optimization of ant foraging behavior. The ACO procedure is illustrated in Figure 1.

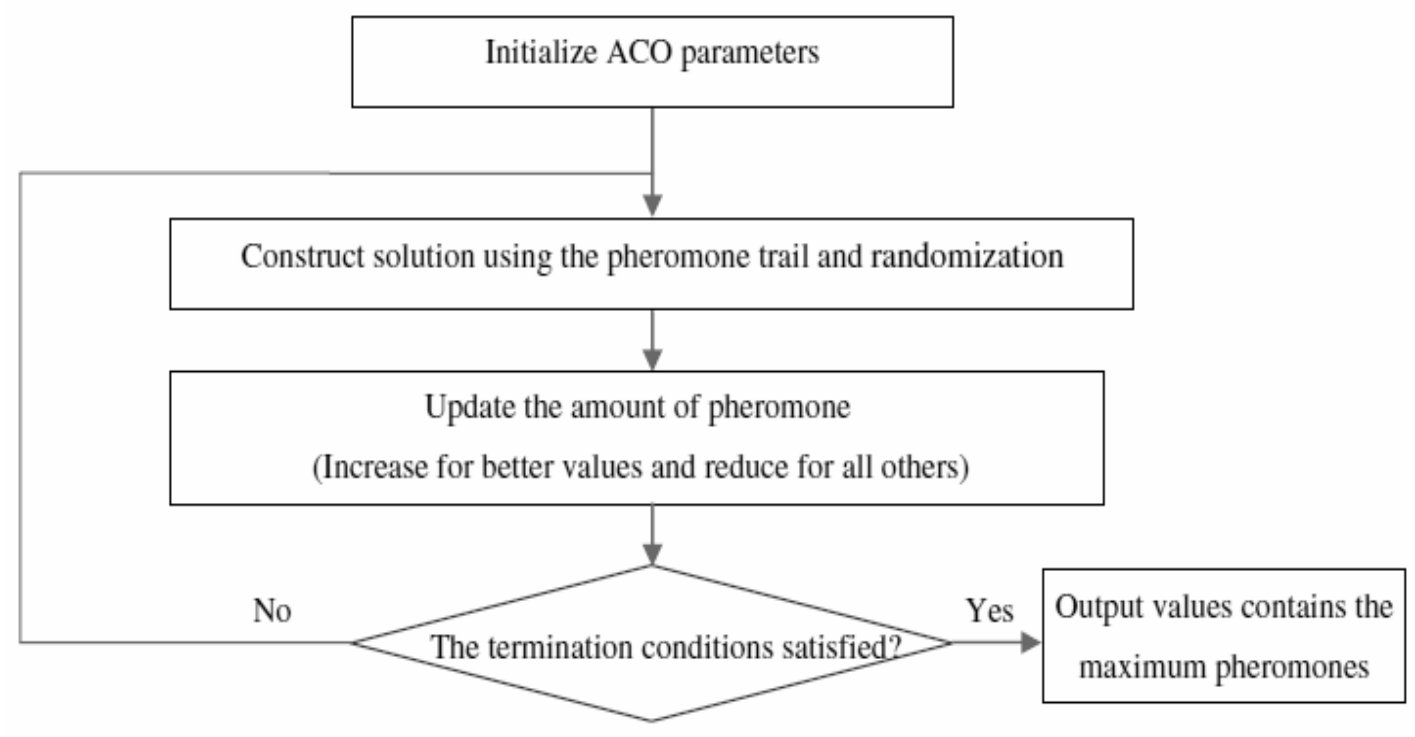

Figure 1. Searching Procedure of the ACO Algorithm 
Ants are insects which live together. Since they are blind animals, they find the shortest path from nest to food with the aid of pheromone. The pheromone is the chemical material deposited by ants, which serves as critical communication media among ants, thereby guiding the determination of the next movement. On the other hand, ants find the shortest path based on the intensity of pheromone deposited on different paths.

\section{The Proposed HPSACO Algorithm}

\subsection{The Idea of the HPSACO Algorithm}

The PSO algorithm takes on faster convergence speed and dynamic topologies for solving optimization problems, but it was easy to fall into the premature convergence in the evolution. The ACO algorithm can quickly discover the optimal solutions for solving optimization problems, but it exists the long search time for solving complex optimization problems. A novel hybrid optimization (HPSACO) algorithm based on combining collaborative strategy, particle swarm optimization and ant colony optimization is proposed in order to balance the exploration of the initial search solution by using the PSO algorithm and the exploitation of the optimal search solution by using the ACO algorithm. The basic idea of the HPSACO algorithm is to divide the ant colony into several ant subgroups, and the four parameters $(\alpha, \beta, \rho, \gamma)$ of each ant subgroup are regarded as one particle of the PSO algorithm. The PSO algorithm is used to optimize all parameters of all ant subgroups and dynamically adjust the initial pheromone distribution of the ACO algorithm in order to better control the development ability and exploration ability of each ant subgroup. Then the pheromone exchange operation is introduced into each ant subgroup to improve the exploration ability and avoid prematurely converging to the local optimal solution. In here, ant subgroup is divided ant colony into the subgroups with the same number of ants. And each ant subgroup maintains own pheromone matrix and parameters. The information exchange is the exchange operation of the pheromone matrix of each ant subgroup.

\subsection{The Flow of the HPSACO Algorithm}

The flow of the novel hybrid optimization (HPSACO) algorithm based on combining collaborative strategy, particle swarm optimization and ant colony optimization is shown in Figure 2.

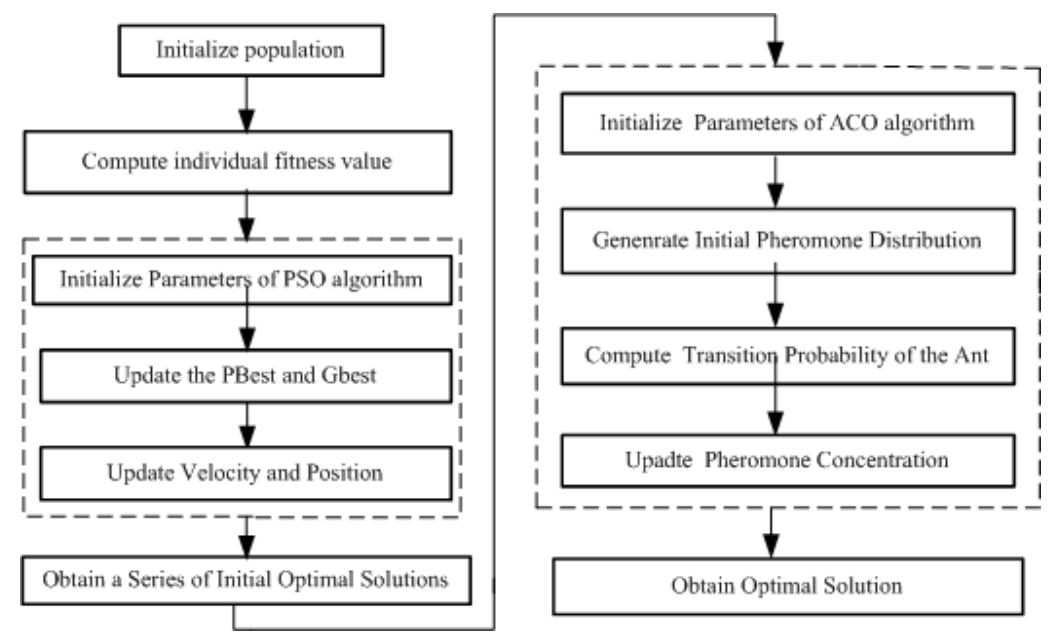

Figure 2. The Flow of the Novel Hybrid Optimization (HPSACO) Algorithm 


\section{Experimental Results and Analysis}

TSP can simply be stated as: a search for the shortest closed tour that visits each city once and only once. The TSP can be described by using a complete directed graph $G=(N, A)$, where $N$ is a set with $n$ cities, $A$ is a set with the arcs. $D=d_{i j}$ is the distance matrix associated with each $\operatorname{arc}(i, j) \in A$. The distance matrix $D$ is symmetric or asymmetric. The essence of the TSP is to find out a shortest closed tour visiting each of the $n=|N|$ cities of $G$. The distances between two cities are independent of the direction of traversing the arcs. That is $d_{i j}=d_{j i}$ for each two cities in symmetric TSP and $d_{i j} \neq d_{j i}$ for each two cities in asymmetric TSP. To define the variables: $x_{i j}=\left\{\begin{array}{ll}1 & \text { if the } \operatorname{arc}(i, j) \text { is in the tour } \\ 0 & \text { otherwise }\end{array}\right.$. Objective function is $z=\min \sum_{i} \sum_{j} d_{i j} x_{i j}$. And the constraints are: $\sum_{i=1}^{n} x_{i j}=1, j=1,2,3, \cdots, n$ and $\sum_{j=1}^{n} x_{i j}=1, i=1,2,3, \cdots, n$.

TSP can simply be stated as: a search for the shortest closed tour that visits each city once and only once. The TSP can be described by using a complete directed graph $G=(N, A)$, where $N$ is a set with $n$ cities, $A$ is a set with the arcs. $D=d_{i j}$ is the distance matrix associated with each $\operatorname{arc}(i, j) \in A$. The distance matrix $D$ is symmetric or asymmetric. The essence of the TSP is to find out a shortest closed tour visiting each of the $n=|N|$ cities of $G$. The distances between two cities are independent of the direction of traversing the arcs. That is $d_{i j}=d_{j i}$ for each two cities in symmetric TSP and $d_{i j} \neq d_{j i}$ for each two cities in asymmetric TSP.

In order to test and verify the performance of the proposed HPSACO algorithm, ten datasets of the traveling salesman problem from TSPLIB are selected in this paper. According to the characteristics of TSPLIB, the distance among any two cities is calculated by the Euclidian distance and then rounded off after the decimal point. These TSP instances are tested by using MATLAB 2010. The PSO algorithm, ACO algorithm and HPSACO algorithm are selected to analyze and compare their optimized performances. The parameters' values of the PSO algorithm, ACO algorithm and HPSACO algorithm could be a complicated problem itself, the change of the parameters could affect the optimum value of the others. So the most reasonable initial values of these parameters are obtained by testing and modifying for some functions. For each TSP, these algorithms are run for 20 times, and the best optimal value and average optimal value are found in here. The obtained initial values of these parameters are shown in Table 1.

Table 1. The Parameters Setting of Three Algorithms

\begin{tabular}{|l|c|c|c|}
\hline \multicolumn{1}{|c|}{ Parameter name } & PSO & ACO & HPSACO \\
\hline Population $\operatorname{Size}(\boldsymbol{M})$ & 200 & N/A & 200 \\
\hline Iteration $\operatorname{Times}\left(\boldsymbol{T}_{\max }\right)$ & 1000 & 1000 & 1000 \\
\hline Inertia Weight $(w)$ & 0.85 & N/A & 0.85 \\
\hline
\end{tabular}




\begin{tabular}{|l|c|c|c|}
\hline Learn Factor & $c_{1}=c_{2}=2$ & N/A & $c_{1}=c_{2}=2$ \\
\hline Ants $(N)$ & N/A & 100 & 100 \\
\hline Pheromone Factor $(\alpha)$ & N/A & 1.0 & 1.0 \\
\hline Heuristic Factor $(\beta)$ & N/A & 2.0 & 2.0 \\
\hline Evaporation Coefficient $(\rho)$ & N/A & 0.05 & 0.05 \\
\hline Pheromone Amount $(Q)$ & N/A & 100 & 100 \\
\hline
\end{tabular}

In our experiment, the scales of ten TSP instances from TSPLIB are from 48 to 14051. For example, eil51, the number of city is 51 . For each TSP, the best optimal value and average optimal value are compared in our simulation experiments. The simulated experiment results are shown in Table 2. The error comparison result for ten TSP is shown Figure 3.

Table 2. The Results of the Simulated Experiments

\begin{tabular}{|c|c|c|c|c|c|c|c|c|c|c|}
\hline \multirow[t]{2}{*}{ Instances } & \multirow{2}{*}{$\begin{array}{c}\text { Optimal } \\
\text { Value }\end{array}$} & \multicolumn{3}{|c|}{ PSO } & \multicolumn{3}{|c|}{ ACO } & \multicolumn{3}{|c|}{ HPSACO } \\
\hline & & Best & Avg. & Err.(\%) & Best & Avg. & Err.(\%) & Best & Avg. & Err.(\%) \\
\hline att 48 & 33522 & 33734 & 33982 & 0.63 & 33649 & 33731 & 0.62 & 33524 & 33667 & 0 \\
\hline st70 & 675 & 691.2 & 702.6 & 2.40 & 685.7 & 694.7 & 1.59 & 680.3 & 698.6 & 0.79 \\
\hline eil76 & 538 & 572.3 & 589.1 & 6.38 & 550.7 & 560.4 & 2.36 & 546.2 & 558.1 & 1.52 \\
\hline pr152 & 73682 & 75361 & 75405 & 2.28 & 74689 & 74936 & 1.37 & 74165 & 74654 & 0.66 \\
\hline gil262 & 2378 & 2513 & 2486 & 5.68 & 2463 & 2495 & 3.57 & 2413 & 2468 & 1.47 \\
\hline rd400 & 15281 & 16964 & 17024 & 11.01 & 16581 & 16834 & 8.51 & 16067 & 16513 & 5.14 \\
\hline pr1002 & 259045 & 278923 & 279755 & 7.67 & 269758 & 271043 & 4.14 & 267998 & 269789 & 3.46 \\
\hline d1291 & 50801 & 53912 & 54104 & 6.12 & 52942 & 53249 & 4.21 & 52868 & 52951 & 4.07 \\
\hline nl4461 & 182566 & 199314 & 199492 & 9.17 & 192964 & 194015 & 5.70 & 191352 & 192585 & 4.81 \\
\hline brd14051 & 469385 & 518631 & 519305 & 10.49 & 505734 & 511638 & 7.74 & 498471 & 503594 & 6.20 \\
\hline
\end{tabular}

As can be seen from the Table 2 and Figure, the best optimal value and average optimal value of the proposed HPSACO algorithm are the best for ten datasets of the traveling salesman problem from TSPLIB in the three given algorithms. In addition, for TSP instances att48 and st70, the obtained solutions 33524 and 680.3 are approaching to the best known solutions 33522 and 675 . For larger scale instances, the Table 2 shows that the average results of the proposed HPSACO algorithm is better than the PSO algorithm and ACO algorithm.

The best routes and their costs (their route lengths) TSP instances att 48 and eil76 are found by using the proposed HPSACO algorithm in Figure 4 and Figure 5. 


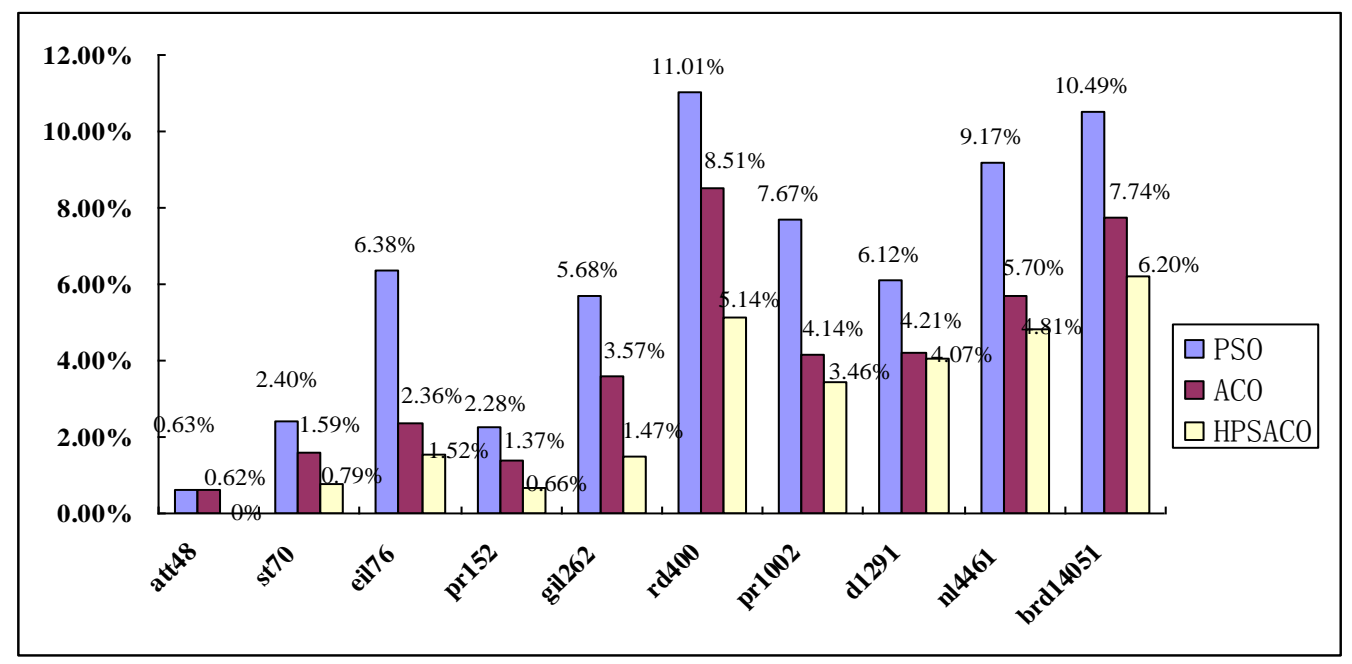

Figure 3. The Error Comparison Result for Ten TSP

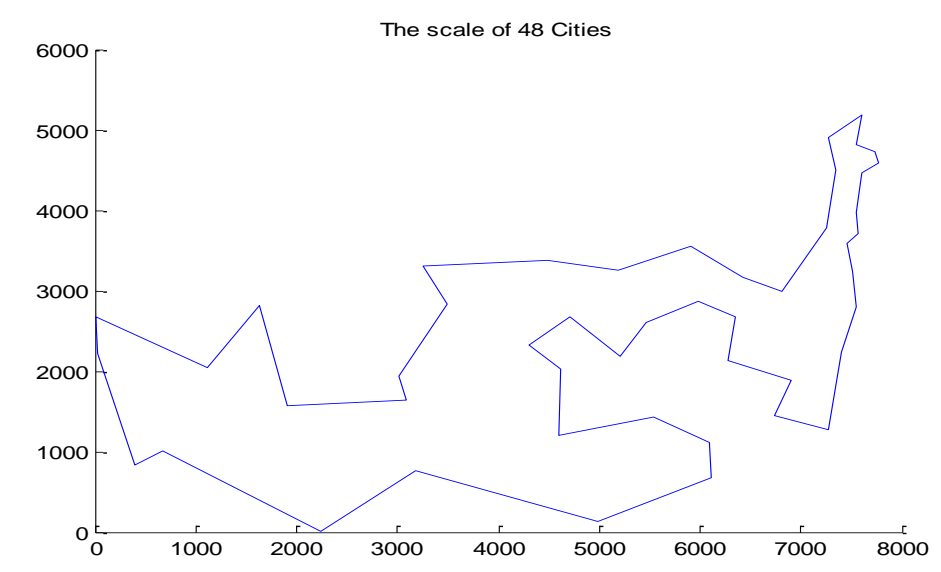

Figure 4. The Best Routes Found by the HPSACO Algorithm for att48

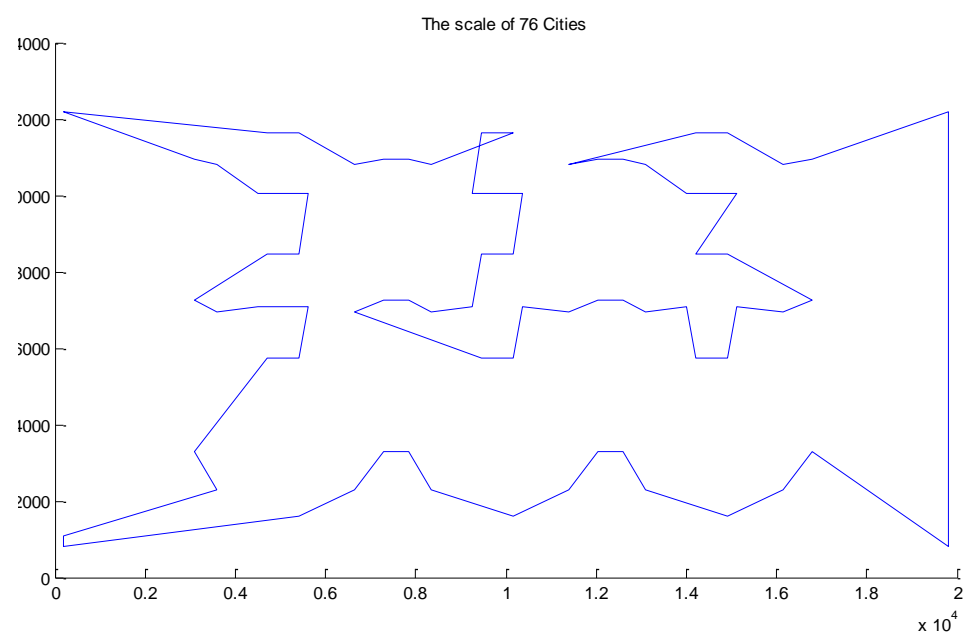

Figure 5. The Best Routes Found by the HPSACO Algorithm for eil76 


\section{Conclusion}

In allusion to the premature and stagnation phenomenon of the ACO algorithm, the operating characteristics of the ACO algorithm is analyzed, and the PSO algorithm and collaborative strategy are introduced into the ACO algorithm in order to propose a novel hybrid optimization (HPSACO) algorithm, which is used to solve the TSP problems. The best solution and average solution quality is improved by using the cooperative evolution scheme of the PSO algorithm and ACO algorithm. Due to the ACO algorithm with the procedure of converging to the global optimum solution. Some benchmark problems from TSPLIB are used to verify the effectiveness of the proposed HPSACO algorithm. The experimental results show that the proposed HPSACO algorithm can obtain the best solution quality and average solution quality by using the proposed algorithm than the PSO algorithm, the ACO algorithm respectively. It has also been shown that the proposed algorithms can solve larger scale optimization problems than those solved using the existing algorithms.

\section{Acknowledgements}

This research was supported by the National Natural Science Foundation of China (U1433124,51475065), Open Project Program of Provincial Key Laboratory for Computer Information Processing Technology, Soochow University (KJS1326), the Open Project Program of Artificial Intelligence Key Laboratory of Sichuan Province (Sichuan University of Science and Engineering) (2014RYJ02).

\section{References}

[1] M. Dorigo and L. M. Gambardella, "Ant colony system: a cooperative learning approach to the traveling salesman problem”, IEEE Transactions on Evolutionary Computation, vol. 1, no. 1, (1997), pp. 53-66.

[2] J. Kennedy and R. C. Eberhart, "Particle Swarm Optimization. Proceeding of the IEEE International Conference on Neural Networks", Piscataway, NJ, (1995), pp. 1942-1948.

[3] G. Acampora, M. Gaeta and V. Loia, "Combining multi-agent paradigm and memetic momputing for personalized and adaptive learning experiences", Computational Intelligence, vol. 27, no. 2, (2011), pp. 141-165.

[4] I. Ellabib, P. Calamai and O. Basir, "Exchange strategies for multiple ant colony system. Information Sciences", vol. 177, no. 5, (2007), pp. 1248-1264.

[5] J. Berger and M. Barkaoui, "A parallel hybrid genetic algorithm for the vehicle routing problem with time windows", Computers\& Operations Research, vol. 31, no. 12, (2004), pp. 2037-2053.

[6] G. Shen and Y. Q. Zhang, "A new evolutionary algorithm using shadow price guided operators", Applied Soft Computing, vol. 11, no. 2, (2011), pp. 1983-1992.

[7] M. Dorigo, L. M. Gambardella, "Ant colonies for the travelling salesman problem”, Biosystems, vol. 43, no. 2, (1997), pp. 73-81.

[8] T. Stutzle and H. Hoos, "The MAX-MIN ant system and local search for the traveling salesman problem", Proceeding of IEEE 4th International Conference on Evolutionary Computation, (1997), pp. 308-313.

[9] Z. J. Lee, C. Y. Lee and S. F. Su, “An immunity-based ant colony optimization algorithm for solving weapon-target assignment problem”, Applied Soft Computing, vol. 2, no. 1, (2002), pp. 39-47.

[10] A. C. Zecchin, A. R. Simpson and H. R. Maier, "Parametric study for an ant algorithm applied to water distribution system optimization", IEEE Transactions on Evolutionary Computation, vol. 9, no. 2, (2005), pp. 175-191.

[11] S. L. Ho and S. Y. Yang, "A computationally efficient vector optimizer using ant colony optimizations algorithm for multobjective designs", IEEE Transactions on Magnetics, vol.44, no. 6, (2008), pp. 1034-1037.

[12] R. S. Chang, J. S. Chang and P. S. Lin, "An ant algorithm for balanced job scheduling in grids", Future Generation Computer System, vol. 25, no. 1, (2009), pp. 20-27. 
[13] M. J. Meena, K. R. Chandran, A. Karthika and A. V. Samuel, "An enhanced ACO algorithm to select features for text categorization and its parallelization", Expert Systems with Applications, vol. 39, no. 5, (2012), pp. 5861-5871.

[14] K. Shima and N. Hossein, "An advanced ACO algorithm for feature subset selection", Neurocomputing, vol. 147, no. 5, (2015), pp. 271-279.

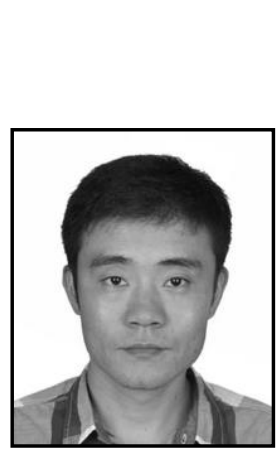

\section{Author}

Hao Jia, Engineer, he received the Master degree in electrical engineering from Dalian Jiaotong University in 2005, Dalian ,China. The main research directions: Artificial Intelligence, Electrical Engineering. 
International Journal of Hybrid Information Technology Vol.8, No.2 (2015) 